\title{
The demographic and clinical characteristics of an Italian population of adult outpatients with chronic cough
}

\author{
Giacomo Campi ${ }^{1} \cdot$ Marianna Noale $^{2}\left(\mathbb{D} \cdot\right.$ Alessio Fabbrizzi $^{1} \cdot$ Federico Lavorini $^{3} \cdot$ Stefania Maggi ${ }^{2} \cdot$ Giovanni Fontana $^{3}$
}

Received: 7 October 2019 / Accepted: 23 December 2019

(c) Springer Nature Switzerland AG 2020

\begin{abstract}
Background Chronic cough is a major health problem worldwide and patients are best managed in specialised tertiary centres. Little information is available on the characteristics of chronic cough patients in several European countries, including Italy. Aims We report on the demographic, anthropometric and clinical features of a large Italian population of adult chronic cough outpatients (about 1200), who were referred to a specialised clinic in Florence, Italy, from 2008 to 2018.

Methods Demographic, environmental, lifestyle and clinical information was collected at enrolment by means of a customdesigned electronic questionnaire that only allowed for uniform responses. A subjective measure of cough-related discomfort (cough score) was also obtained using a modified Borg Scale. A multivariable logistic regression model was defined to identify the patients' characteristics associated with the cough score.

Results The characteristics of the examined population ( $n=1204$ outpatients) were strikingly similar to those described elsewhere. Female patients outnumbered the males $[n=847$ females, $(70.0 \%)]$; both females and males displayed the same average cough score. The median age of outpatients was 61 (quartile $1=48$; quartile $3=70$ ) years; age and cough duration were unrelated to the cough score. Nasal obstruction, coughing during consultation, coughing during meals, throat clearing and the presence of respiratory abnormalities were correlated with the degree of discomfort caused by coughing.

Discussion The features of chronic cough patients are similar worldwide. The process of cough reflex hypersensitisation may soothe sex-related perceptual differences, leading to similar levels of discomfort.

Conclusions There seem to be clinical indicators that help in assessing the level of cough-related discomfort.
\end{abstract}

Keywords Chronic cough $\cdot$ Cough score $\cdot$ Cough-related discomfort

\section{Introduction}

It is well known that cough represents one of the commonest complaints worldwide. A substantial degree of morbidity is associated with chronic cough, generally defined as a cough

Electronic supplementary material The online version of this article (https://doi.org/10.1007/s40520-019-01464-4) contains supplementary material, which is available to authorized users.

Marianna Noale

marianna.noale@in.cnr.it

1 Postgraduate School of Respiratory Medicine, University of Florence, Florence, Italy

2 Italian National Research Council (CNR), Neuroscience Institute, Aging Branch, Padua, Via Giustiniani 2, 35128 Padua, Italy

3 Department of Experimental and Clinical Medicine, University of Florence, Florence, Italy being the only or prevalent symptom lasting for at least 8 weeks in the absence of obvious radiographic evidence of lung disease [1-3]. Although chronic cough may characterise several respiratory and extra-respiratory disorders such as asthma, pulmonary fibrosis, chronic obstructive pulmonary disease and gastro-oesophageal reflux, many chronic cough patients have virtually no accompanying symptoms, so that the underlying causes remain difficult to detect. Several international guidelines $[1,2]$, published by different scientific assemblies, are currently available for the assessment of chronic cough patients, and it seems desirable for patients with chronic cough to be referred to a specialist clinic for optimal evaluation [3]. Nonetheless, the number of specialised centres is limited in Italy and indeed the Cough Clinic at the Careggi University Hospital in Florence appears to be the only institutional unit specifically devoted to the management of adult chronic cough patients. 
This manuscript reports on the demographic and clinical features of an unselected sample of Italian adult outpatients with chronic cough who were referred to the Careggi Cough Clinic; in addition, it aims to determine the association between the patients' clinical characteristics and a subjective measure of the discomfort caused by their cough.

\section{Methods}

We enrolled all adult outpatients with chronic cough (i.e. lasting longer than 2 months) [1-3] who were consecutively referred to our Cough Clinic between 2008 and 2018. The exclusion criteria were patients with a history of recent ( $<4$ weeks) respiratory infection and/or evidence of active lesions documented by a recent (within 2 months) chest $\mathrm{X}$-ray. All procedures complied with the Helsinki Declaration and the study was approved by the local Institutional Review Board (OSS_14131). The study followed the guidelines for observational studies [4].

\section{Data collection}

By using a custom-designed electronic questionnaire, we collected a set of demographic, environmental, lifestyle and clinical information for each patient at enrolment. The questionnaire comprised several distinct items and was conceived to only allow for unified responses, thus making its administration completely independent of the operator. A subjective measure of cough-related discomfort (hereafter termed "cough score") was also obtained by using a 0-9 modified Borg Scale (see supplement). The time required for the questionnaire administration, cough score and clinical assessment at consultation was $40-50 \mathrm{~min}$. Additional details of the questionnaire and cough score assessment are available as supplementary material.

\section{Statistical analysis}

Categorical variables were presented as numbers with the corresponding percentages, whereas continuous variables were summarised through either the mean and standard deviation (SD) if normally distributed (Shapiro-Wilk test), or the median and Q1-Q3 quartiles. Based on its distribution, the cough score was dichotomised into $<3$ vs. $\geq 3$ ("the cough does not bother me at all or causes only slight discomfort" vs. "the cough bothers me or causes me moderate, severe, very severe, or extremely severe discomfort", see Supplement for additional details). The relationships between the dichotomised cough score and the patients' characteristics were assessed by using Fisher's exact test, the Chi-squared test and the Wilcoxon rank-sum test where appropriate. A multivariable logistic regression model was defined with the dichotomised cough score as the outcome. A stepwise selection procedure of covariates was used, with $p \leq 0.20$ to enter the model and $p \leq 0.10$ to stay in the model [5]. Linearity assumption for quantitative variables and interactions among independent variables were also evaluated in the model.

All the statistical tests were two-tailed and $p$ values $<0.05$ were considered significant. All the analyses were performed using the SAS 9.4 statistical software (SAS Institute, Cary, NC, USA).

\section{Results}

The general and dichotomised demographic, anthropometric and clinical characteristics of the patient cohort $(n=1204$, $70.4 \%$ females) are reported in Table 1 .

In the general population, the median age was $61(48-70)$ years, with no significant difference between males or females (Fig. $1 ; p=0.8154$ ). The overall mean body mass index (BMI) was $25.8 \pm 4.4 \mathrm{~kg} / \mathrm{m}^{2}$, with more than half of the sample overweight or obese (BMI $\geq 25 \mathrm{~kg} / \mathrm{m}^{2}$ ). Less than $10 \%$ of patients were smokers, with average daily smoking of ten cigarettes (5-20). Only 21 patients (9 women) with previously undiagnosed COPD were included in the study. The median duration of cough was 3 years $(1-10)$ and distribution was right-skewed, with more than $40 \%$ of patients referring for a cough lasting 5 years or longer. As shown in Fig. 2, the overall median cough score was 5 (3-7) with no significant difference between males or females $(p=0.4733)$. The majority of patients (about 59\%) were not on any treatment for their cough at the time of consultation. Treatment taken by the remaining patients at the time of consultation—or during the period immediately preceding — mostly included anti-reflux drugs, asthma medications, antibiotics and over-the-counter antitussives.

Patients with a cough score $\geq 3$ had more frequent accompanying symptoms and clinical signs than those with $\mathrm{a}<3$ cough score (Table $1 ; p<0.05$ for exertional wheezing, night cough, cough under effort, post-nasal drip (PND) sensation, voice changes, frequent heartburn, regurgitation, globus sensation, cough on walking, bending, talking/phoning, eating, after meals, evoking epigastric pain; cough worsened by changes in body position, strong smells and upper airway infections). Notably, among patients with $<3$ cough score, with respect to those with a score $\geq 3$, cough ensuing during consultation $(62.9 \%$ vs. $23 \%$, respectively; $p<0.0001)$, evoking epigastric pain $(26.1 \%$ vs. $13.9 \% ; p=0.0069)$ as well as the induction of deflation cough $(40.8 \%$ vs. $24.7 \%$, respectively; $p=0.0025$ ) was more frequently observed [6, 7]. The use of ACE inhibitors was reported by 82 outpatients; the percentage of patients using ACE inhibitors with a cough score $\geq 3$ was not significantly different from that among patients with $\mathrm{a}<3$ cough score $(p=0.6404)$. 
Table 1 Overall and dichotomised by cough score characteristics of patients at enrolment

\begin{tabular}{|c|c|c|c|c|}
\hline & Overall $(n=1204)$ & $\mathrm{CS}<3(n=104)$ & $\mathrm{CS} \geq 3(n=1054)$ & $p$ value \\
\hline Sex, females, $n(\%)$ & $847(70.4)$ & $70(67.3)$ & 759 (70.7) & 0.4733 \\
\hline Age, years, median (Q1, Q3) & $61(48,70)$ & $65(46.5,72.5)$ & $61(48,69)$ & 0.1369 \\
\hline BMI, $\mathrm{kg} / \mathrm{m}^{2}$, median $(\mathrm{Q} 1, \mathrm{Q} 3)$ & $25.8 \pm 4.4$ & $25.8 \pm 4.2$ & $25.8 \pm 4.4$ & 0.8046 \\
\hline Smoking status, $n(\%)$ & & & & 0.3035 \\
\hline Never & $735(61.9)$ & $69(66.4)$ & $661(61.7)$ & \\
\hline Ex & $349(29.4)$ & $24(23.1)$ & $321(29.9)$ & \\
\hline Current & $104(8.8)$ & $11(10.6)$ & $90(8.4)$ & \\
\hline ACE inhibitor use, $n(\%)$ & $82(6.9)$ & $6(5.8)$ & $75(7.0)$ & 0.6404 \\
\hline Seasonal cough, $n(\%)$ & & & & 0.002 \\
\hline Continuous & $728(80.3)$ & $52(67.5)$ & $672(81.4)$ & \\
\hline Periodic & $88(9.7)$ & $16(20.8)$ & $72(8.7)$ & \\
\hline Seasonal & $91(10.0)$ & $9(11.7)$ & $82(9.9)$ & \\
\hline Asthma familiarity, $n(\%)$ & $252(21.9)$ & $17(16.7)$ & $233(22.4)$ & 0.1812 \\
\hline Allergy symptoms, $n(\%)$ & $357(35.2)$ & $31(32.0)$ & $324(35.6)$ & 0.475 \\
\hline Exertional wheezing, $n(\%)$ & $408(34.5)$ & $25(24.0)$ & $378(35.3)$ & 0.0206 \\
\hline Exceptional dyspnoea, $n(\%)$ & $438(37.4)$ & $30(28.9)$ & $404(38.1)$ & 0.0622 \\
\hline Sputum, $n(\%)$ & $314(26.6)$ & $26(25.2)$ & $283(26.5)$ & 0.7907 \\
\hline Night cough, $n(\%)$ & $625(52.7)$ & $45(43.3)$ & $576(53.6)$ & 0.0413 \\
\hline Cough under effort, $n(\%)$ & $441(38.2)$ & $25(24.3)$ & $412(39.4)$ & 0.0025 \\
\hline Cough in cold air, $n(\%)$ & $431(37.3)$ & $30(29.4)$ & $400(38.2)$ & 0.0798 \\
\hline PND sensation, $n(\%)$ & $352(30.1)$ & $19(18.3)$ & $329(31.1)$ & 0.0063 \\
\hline Throat clearing, $n(\%)$ & $897(76.3)$ & $69(66.4)$ & $823(77.4)$ & 0.0111 \\
\hline Voice changes, $n(\%)$ & $620(52.5)$ & $44(42.3)$ & $570(53.3)$ & 0.0326 \\
\hline Frequent heartburn, $n(\%)$ & $254(21.4)$ & $14(13.5)$ & $237(22.1)$ & 0.0395 \\
\hline Indigestion, $n(\%)$ & 399 (33.9) & $29(28.2)$ & $366(34.4)$ & 0.2033 \\
\hline Frequent regurgitation, $n(\%)$ & $236(19.9)$ & $12(11.5)$ & $222(20.8)$ & 0.0248 \\
\hline Belching/burping, $n(\%)$ & $466(39.3)$ & $36(34.6)$ & $426(39.7)$ & 0.3105 \\
\hline Globus sensation, $n(\%)$ & $536(45.9)$ & $26(25.5)$ & $509(48.1)$ & $<0.0001$ \\
\hline Cough on walking, $n(\%)$ & $653(56.1)$ & $37(35.6)$ & $611(57.9)$ & $<0.0001$ \\
\hline Cough on bending, $n(\%)$ & $310(26.7)$ & $16(15.7)$ & $290(27.5)$ & 0.0096 \\
\hline Cough on talking/phone, $n(\%)$ & 799 (67.7) & $43(41.8)$ & $753(70.3)$ & $<0.0001$ \\
\hline Cough on eating, $n(\%)$ & $314(26.7)$ & $16(15.4)$ & $295(27.7)$ & 0.0066 \\
\hline Cough after meals, $n(\%)$ & $400(34.0)$ & $24(23.1)$ & $375(35.2)$ & 0.0132 \\
\hline Deflation cough, $n(\%)$ & $385(39.2)$ & $23(24.7)$ & $360(40.8)$ & 0.0025 \\
\hline Cough during visit, $n(\%)$ & $622(59.0)$ & $23(23.0)$ & $596(62.9)$ & $<0.0001$ \\
\hline Respiratory signs, $n(\%)$ & $370(32.1)$ & $26(25.5)$ & $339(32.5)$ & 0.1453 \\
\hline Evoked epigastric pain, $n(\%)$ & $285(25.1)$ & $14(13.9)$ & $267(26.1)$ & 0.0069 \\
\hline \multicolumn{5}{|l|}{ Cough worsened by, $n(\%)$} \\
\hline $\begin{array}{l}\text { Certain foods/beverages } \\
\text { (including alcohol, coffee, } \\
\text { cold drinks) }\end{array}$ & 199 (19.6) & $13(16.1)$ & $186(20.1)$ & 0.384 \\
\hline Climate changes & $250(24.7)$ & $15(13.6)$ & $234(25.2)$ & 0.1784 \\
\hline Changes in body position & $313(30.9)$ & $12(14.8)$ & $301(32.5)$ & 0.001 \\
\hline $\begin{array}{l}\text { Environmental exposure (dust, } \\
\text { tobacco smoke, smog) }\end{array}$ & $195(19.2)$ & $10(12.4)$ & $183(19.7)$ & 0.1047 \\
\hline Laughing & $157(15.5)$ & $13(16.1)$ & $143(15.4)$ & 0.8818 \\
\hline Stress & $95(9.4)$ & $8(9.9)$ & $85(9.2)$ & 0.833 \\
\hline Strong smells & $320(31.6)$ & $16(19.8)$ & $304(32.8)$ & 0.015 \\
\hline Upper airway infections & $122(12.0)$ & $19(23.5)$ & $103(11.1)$ & 0.0011 \\
\hline
\end{tabular}

$A C E$ angiotensin converting enzyme, $C S$ cough score, $P N D$ post nasal drip 


\section{Age distribution by sex}

- Females amales

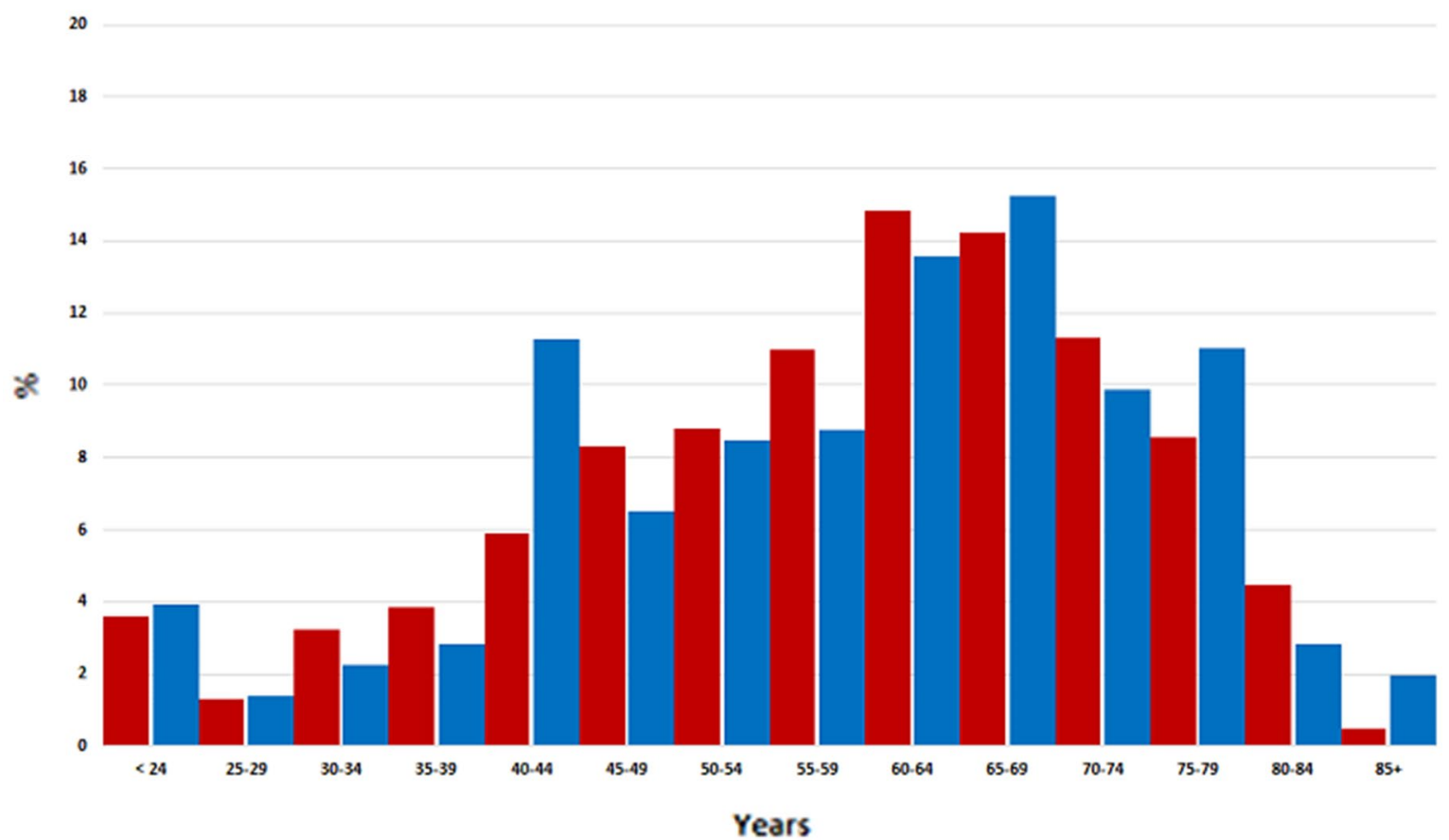

Fig. 1 Frequency distribution of age (years) by gender at enrolment

\section{Cough Score (0-9) by sex}

-Females = Males

25

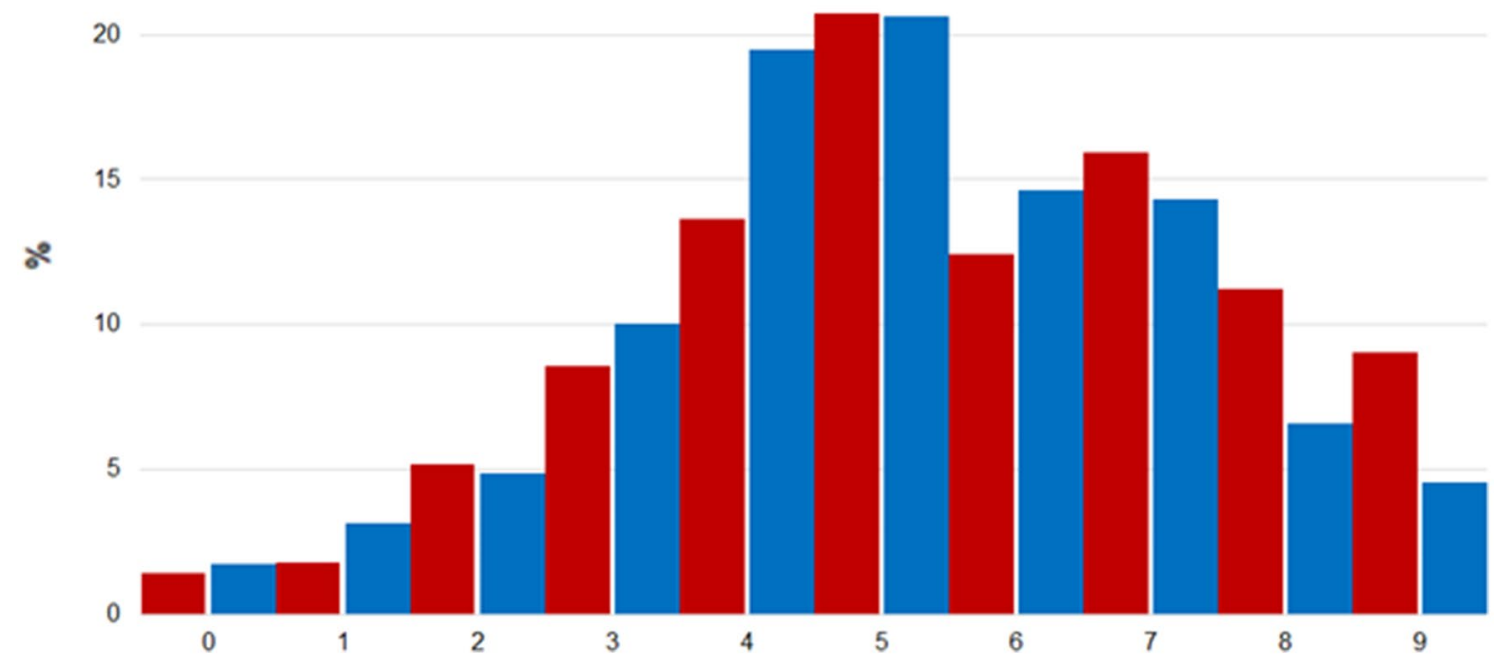

Fig. 2 Frequency distribution of cough score by gender at enrolment 
Table 2 Multivariable logistic regression model with outcome of dichotomised cough score $(\mathrm{CS})$ at enrolment $(\mathrm{CS}<3$ vs. $\mathrm{CS} \geq 3)$

\begin{tabular}{lllr}
\hline & OR & $95 \%$ CI & $p$ value \\
\hline Sex (females vs. males) & 1.11 & $0.56-2.20$ & 0.7607 \\
Age ( $\geq 65$ vs. $<65$ years) & 0.62 & $0.32-1.18$ & 0.1423 \\
Cough duration (years) & 1.03 & $0.98-1.09$ & 0.1861 \\
Nasal obstruction & 2.45 & $1.13-5.35$ & 0.0241 \\
Throat clearing & 2.2 & $1.11-4.38$ & 0.0239 \\
Cough on waking up & 1.79 & $0.95-3.39$ & 0.0731 \\
Cough during eating & 3.11 & $1.32-7.33$ & 0.0095 \\
Cough during visit & 5.88 & $2.86-12.1$ & $<0.0001$ \\
Respiratory signs & 2.29 & $1.12-4.72$ & 0.0241 \\
Drugs at admission: antacid & 0.39 & $0.19-0.8$ & 0.0104 \\
Drugs at admission: antihistamine & 0.28 & $0.11-0.72$ & 0.0080 \\
Drugs at admission: PPI & 0.60 & $0.31-1.18$ & 0.1389 \\
\hline
\end{tabular}

PPI proton pump inhibitors

The results of the multivariable logistic regression analysis (Table 2) showed that the patients' characteristics most frequently associated with a cough score $\geq 3$ were stuffy nose (odds ratio (OR) $2.45,95 \%$ confidence interval (CI) $1.13-5.35 ; p=0.0241)$, throat clearing (OR 2.2, 95\% CI $1.11-4.38 ; p=0.0239$ ), coughing on eating (OR $3.11,95 \%$ CI 1.32-7.33; $p=0.0095$ ), coughing during the visit (OR $5.88,95 \%$ CI $2.86-12.1 ; p<0.0001)$ and the presence of respiratory signs (e.g. wheezing and/or crackles) on clinical examination (OR 2.29, 95\% CI 1.12-4.72; $p=0.0241$ ). Conversely, antacid and antihistamine drug use was associated with a lower cough score (Table 2; OR 0.39, 95\% CI $0.19-0.80, p=0.0104$; and $\mathrm{OR}=0.28,95 \%$ CI $0.11-0.72$, $p=0.0080$, respectively). Age, sex and cough duration were all unrelated to the cough score at enrolment $(p>0.05)$.

\section{Discussion}

This is the first report on the demographic, anthropometric and clinical characteristics of an unselected group of adult chronic cough outpatients referring to a tertiary care centre in Italy. It confirms that age and sex distribution in this large group of patients overlaps that reported in previous population studies performed in other countries, despite obvious cultural, environmental and behavioural differences [8]. Accordingly, we documented a preponderance of females suffering from a primary complaint of chronic cough in our study $[9,10]$. This finding is in keeping with the notion of females having a heightened cough reflex sensitivity as revealed by inhalational challenges [11-14] and in response to treatment with ACE inhibitors [15]. However, the fact that the distribution of cough scores was virtually identical in males and females indicates that gender is unrelated to the magnitude of the perceived discomfort caused by the cough. This finding seems to be in contrast with the observations derived from a recent functional magnetic-resonance study showing a much greater activation in the somatosensory cortex of healthy females than in males during an inhalational capsaicin challenge [8]. Since chronic cough can be regarded as a clinical syndrome characterised by neuronal hypersensitivity [16], it may be that the processes underlying hypersensitisation of the cough reflex could have blunted the natural sex-related perceptual differences, leading to comparable degrees of perceived discomfort between male and female chronic coughers.

We found that the mean cough duration in our population was about 3 years, although many patients reported a cough lasting longer than 25 years (data not shown). Nonetheless, cough duration was unrelated to the magnitude of the perceived discomfort. A likely explanation for this finding may be habituation to the burden imposed by the cough. Over time, patients reportedly develop different strategies to cope with their disability and, conceivably, the better their strategy the less is the discomfort.

This study has some limitations. First, these patients are not representative of all patients with chronic cough. Indeed, a patient usually receives medical attention from a general practitioner, then from a specialist and, eventually, from a tertiary care centre. Consequently, most patients with chronic cough due to common causes, such as chronic obstructive pulmonary disease, heart failure or use of ACE inhibitors, are treated in other settings. The patients being treated at the Careggi Cough Clinic are therefore those with undiagnosed causes or who have been unsuccessfully treated for selected causes.

Second, the results do not give a reliable picture of the epidemiology of chronic cough in Italy. Indeed, the vast majority of the patients (about 80\%) were referred from areas close to the Florentine clinic, whereas only a minority were from regions outside Tuscany. Nonetheless, the fact that the demographic and clinical characteristics of the patients included in this survey were comparable to those of studies performed in other world areas [8] points to the possibility that chronic cough phenotypes are similar all over the world. If this is the case, the possibility that chronic cough patients from other Italian areas have comparable characteristics also seems likely.

Another limitation may be the lack of a more objective evaluation of the burden of cough in our patients. Common methods for the quantitative analysis of cough severity include objective recordings of cough frequency and administration of cough-related quality-of-life questionnaires, such as the Leicester Cough Questionnaire (LCQ) [17]. In real life settings, objective cough frequency recording techniques are difficult for several reasons, mainly the poor availability of validated and time-efficient devices on the market. On the 
other hand, administration of the LCQ would be difficult since the tool has not been validated in Italy and it would extend the duration of each consultation somewhat beyond the acceptable time limits. Interestingly, we found a strong association ( $\mathrm{OR}=5.88)$ between "coughing during the visit" and the cough score. In short, patients who coughed during the consultation had a sixfold higher possibility of a cough score $>3$. We suggest that coughing during visit could be taken as an additional indicator of the magnitude of the disturbance caused by coughing in chronic cough patients.

In conclusion, this is the first report on the characteristics of a large population of chronic cough outpatients who were referred to a specialised cough clinic in Italy. Notably, these characteristics appear to mirror those described in other population studies performed worldwide [8]. Our findings are therefore in keeping with the possibility that chronic cough patients have a single common clinical entity. Finally, based on a purely clinical approach, we have identified useful clinical indicators that may help to assess cough severity. Further studies are needed to ascertain whether these clinical indicators could also be useful in longitudinal studies aimed at determining the prevailing causes of chronic cough and the outcomes of therapeutic interventions.

Acknowledgements This work was funded by an unrestricted grant from Merck. We wish to thank all those physicians and post-graduate students who participated in the data collection throughout the years.

Author contributions GC, FL, AF and GAF conceived and designed the study, interpreted the data and drafted the manuscript, which was critically revised by all authors. SM and MN analysed the data. All authors had access to the complete study data and had a say in the manuscript preparation, approval of the final version and the decision to submit for publication. GAF is the guarantor.

\section{Compliance with ethical standards}

Conflict of interest On behalf of all authors, the corresponding author states that there is no conflict of interest.

Ethical approval All procedures performed in this study involving human participants were in accordance with the ethical standards of the institutional research committee (Ethics Committee of the Azienda Ospedaliero-Universitaria Careggi, Florence, Italy; OSS_14131) and with the 1964 Helsinki declaration and its later amendments or comparable ethical standards.

Informed consent All the participants signed an informed consent form.

\section{References}

1. Pratter MR, Brightling CE, Boulet LP et al (2006) An empiric integrative approach to the management of cough: ACCP evidence-based clinical practice guidelines. Chest 129:222S-231S

2. Morice AH, Fontana GA, Belvisi MG et al (2007) ERS guidelines on the assessment of cough. Eur Respir J 29:1256-1276

3. Morice AH, McGarvey L, Pavord I (2006) Recommendations for the management of cough in adults. Thorax 61:i1-i24

4. von Elm E, Altman DG, Egger M, Pocock SJ, Gøtzsche PC, Vandenbroucke JP; STROBE Initiative (2007) Strengthening the reporting of observational studies in epidemiology (STROBE) statement: guidelines for reporting observational studies. BMJ 335:806-808

5. Hosmer DW, Lemeshow S, Sturdivant RX (2013) Applied logistic regression, 3rd edn. Wiley, Hoboken

6. Lavorini F, Fontana GA, Chellini E et al (2010) Respiratory expulsive efforts evoked by maximal lung emptying. Chest 140:690-696

7. Lavorini F, Chellini E, Bigazzi F et al (2016) The clinical value of deflation cough in chronic coughers with reflux symptoms. Chest 149:1467-1472

8. Morice AH, Jakes AD, Faruqi S et al (2014) A worldwide survey of chronic cough: a manifestation of enhanced somatosensory response. Eur Respir J 44:1149-1155

9. Morice AH (2002) Epidemiology of cough. Pulm Pharmacol Ther 15:253-259

10. Bende M, Millqvist E (2012) Prevalence of chronic cough in relation to upper and lower airway symptoms; the Skövde populationbased study. Front Physiol 3:25

11. Rostami-Hodjegan A, Abdul-Manap R, Wright CE et al (2001) The placebo response to citric acid-induced cough: pharmacodynamics and gender differences. Pulm Pharmacol Ther 14:315-319

12. Kelsall A, Decalmer S, McGuinness K et al (2009) Sex differences and predictors of objective cough frequency in chronic cough. Thorax 64:393-398

13. Fujimura M, Sakamoto S, Kamio Y et al (1990) Sex difference in the inhaled tartaric acid cough threshold in non-atopic healthy subjects. Thorax 45:633-634

14. Dicpinigaitis PV, Rauf K (1998) The influence of gender on cough reflex sensitivity. Chest 113:1319-1321

15. Semple PF (1995) Putative mechanisms of cough after treatment with angiotensin converting enzyme inhibitors. J Hypertens Suppl. 13:S17-21

16. Chung KF, McGarvey L, Mazzone S (2016) Chronic cough and cough hypersensitivity syndrome. Lancet Respir Med 4:934-935

17. Birring SS, Prudon B, Carr AJ et al (2003) Development of a symptom specific health status measure for patients with chronic cough: Leicester Cough Questionnaire (LCQ). Thorax 58:339-343

Publisher's Note Springer Nature remains neutral with regard to jurisdictional claims in published maps and institutional affiliations. 